\title{
The Overexpression of Kinesin Superfamily Protein 2A (KIF2A) was Associated with the Proliferation and Prognosis of Esophageal Squamous Cell Carcinoma
}

This article was published in the following Dove Press journal:

Cancer Management and Research

\author{
Demao Li' \\ Huijie Sun ${ }^{2}$ \\ Linglei Meng ${ }^{3}$ \\ Deshang $\mathrm{Li}^{4}$ \\ 'Department of Thoracic Surgery, Xingtai \\ People's Hospital, Xingtai City, Hebei \\ 054000, People's Republic of China; \\ ${ }^{2}$ Department of Pharmacy, Xingtai \\ Medical College, Xingtai City, Hebei \\ 054000, People's Republic of China; \\ ${ }^{3}$ Department of CT/MR, Xingtai People's \\ Hospital, Xingtai City, Hebei 054000, \\ People's Republic of China; ${ }^{4}$ Department \\ of Laboratory, Xingtai People's Hospital, \\ Xingtai City, Hebei 054000, People's \\ Republic of China
}

\begin{abstract}
Aim: Kinesin family member $2 \mathrm{~A}$ (KIF2A) is a member of the kinesin-13 superfamily protein. KIF2A played a role in the development of many tumors. However, the role of KIF2A in esophageal squamous cell carcinoma (ESCC) remains unclear. In this study, we aimed to investigate the role of KIF2A in ESCC.
\end{abstract}

Methods: We used bioinformatics analysis to study the expression levels and prognosis of KIF2A in ESCC and normal tissues. We also used our own samples to verify the results by immunohistochemistry. Then, the biological functions of KIF2A in ESCC was studied by cell experiments and animal experiments.

Results: Both the TCGA database and our samples showed that KIF2A was relatively highly expressed in ESCC tissues and was significantly associated with disease-free survival ( $\mathrm{P}=0.037)$ in TCGA database. Colony formation assay, CCK8 and Western blotting results showed that knockdown of KIF2A can significantly reduce colony forming ability and proliferation ability. The results of animal experiments showed that knocking down KIF2A can significantly reduce the tumor volume of mice.

Conclusion: KIF2A might be used as a prognostic factor for ESCC, and knockdown of KIF2A could inhibit ESCC proliferation in vitro and in vivo, respectively. KIF2A could serve as a potential prognostic biomarker and therapeutic target for future ESCC.

Keywords: esophageal squamous cell carcinoma, kinesin superfamily protein 2A, ESCC, KIF2A, proliferation

\section{Introduction}

Esophageal cancer is very difficult to cure around the world. ${ }^{1-3}$ The most representative of these is China, which accounts for about $50 \%$ of global cases of esophageal cancer. The main pathological type is esophageal squamous cell carcinoma (ESCC). ${ }^{4,5}$ During the last decade, the development of multiple therapeutic strategies for esophageal cancer including radical surgery, chemotherapy, and radiotherapy has improved the prognosis of patients with this malignancy; ${ }^{6-8}$ however, the 5-year survival rate of esophageal cancer patients is less than $30 \%$. Thus, there is an urgent need to further understand the molecular mechanisms underlying ESCC tumorigenesis for the treatment of ESCC.

In recent years, studies had successively identified the kinesin 13 family, including the kinesin family member $2 \mathrm{~A}$ (KIF2A), etc. ${ }^{9-11}$ They are related to the spindle
Correspondence: Deshang Li

Department of Laboratory, Xingtai People's Hospital, 16 Hongxing Street,

Xingtai City, Hebei 054000, People's

Republic of China

Email Idsashj@sina.com 
and are essential for precise chromosome segregation in mitotic and meiotic cell cycles. ${ }^{10,12}$ Among them, KIF2A acts as a microtube minus-end depolymerization motor, which is an essential component in assembling ordinary bipolar spindles. ${ }^{13}$ In recent years, some evidence suggests that KIF2A may be involved in carcinogenesis. ${ }^{14-16}$ For example, aberrant expression of KIF2A is associated with the prognosis of gastric cancer, ${ }^{17}$ breast cancer, ${ }^{15}$ and glioma. ${ }^{18}$ However, there is no clear study on the role of KIF2A in ESCC and its mechanism.

In our study, we analyzed the protein expression level of KIF2A in ESCC patients, revealing that overexpression of KIF2A is associated with poor clinical outcome. We have revealed that knocking down KIF2A could significantly inhibit the proliferation of ESCC in both cell lines and mice. Because KIF2A can promote the proliferation of ESCC tumors and is associated with the prognosis of ESCC.

\section{Methods}

\section{Bioinformatics Analysis}

The mRNA-seq data for ESCC patients herein is data from the Cancer Genome Atlas (TCGA). ${ }^{19}$ Download at: http:// firebrowse.org/ (Broad Institute TCGA Genome Data Analysis Center).

\section{Cells Culture}

The human ESCC cell lines CaEs-17 and EC-109 used in this study were purchased from Keygen Biotech (Nanjing, China). Among them, CaEs-17 cells were cultured in Dulbecco's modified Eagle medium (DMEM) (Biological Industries), while EC-109 cells were cultured in RPMI-1640 medium (Biological Industries). Both media were then supplemented with $10 \%$ fetal bovine serum (FBS) (Biological Industries), $100 \mathrm{U} / \mathrm{L}$ penicillin and $0.1 \mathrm{mg} / \mathrm{mL}$ streptomycin (Biological Industries). We have to keep the cells in a humidified incubator containing $5 \% \mathrm{CO}_{2}$ at $37^{\circ} \mathrm{C}$ for a long time.

\section{Immunohistochemistry (IHC)}

The human ESCC organizations used in this article are taken from patients who are self-hospital, and these are surgically cut tissues. The ESCC tissue was first deparaffinized, rehydrated in successively different concentrations of ethanol bath, placed in a pressure cooker and soaked in citrate buffer, and the antigen was recovered after approximately 30 minutes. KIF2A was then immunostained with a polyclonal antibody (ab197988, Abcam plc, Cambridge,
UK) at a 1:200 dilution. Enzymatic detection of antibodies. The results were centrally reviewed and scored by two independent pathologists who were blinded. The scores were divided into four grades which are $0,1,2,3$ respectively. The use of human samples in this study was approved by the Ethics Committee of Xingtai people's hospital, in accordance with the Declaration of Helsinki. The research involving human participants experiments had been approved by our hospital and our equivalent committee. The participants provided their written informed consents to participate in this study.

\section{Transfection}

Commercialized Ready-to-package AAV shRNA clones of KIF2A was purchased. The target sequences are as follows: sh1: 5'-GGAATGGCATCCTGTGAAA-3' and sh2: 5'--C ACCGGCAAAGAGATTGACCTGGT-3'. RNA iMAX reagent was used to transfect to the ESCC cells. Negative Control group, which transfected with scrambled sequence. Transfections were conducted using lipofectamine 2000 (11668019, Invitrogen, Carlsbad, CA, USA). Stable knockdown of ESCC cells was obtained by its shRNA lentivirus infection and selection was performed by puromycin supplementation and used in animal experiments.

\section{Quantitative Polymerase Chain Reaction (qPCR)}

TRIZOL reagent was added to the cultured cells to extract total RNA. The isolated RNA is then reverse transcribed into cDNA. The cDNA was then subjected to qPCR analysis. In these two-steps we used TransScript Green TwoStep qPCR SuperMix (Transgen biotech, beijing, China). Our results are all based on $\beta$-actin as an internal reference. The primers were as follows: KIF2A sense 5'- GCC TTTGATGACTCAGCTCC-3', reverse 5'- TTCCTGA AAAGTCACCACCC-3'; $\beta$-actin sense 5'-TGACGTGG ACATCCGCAAAG-3', reverse 5'-CTGGAAGGTGGAC AGCGAGG-3'. The results were expressed as mean \pm standard deviation (SD). The relative quantification of KIF2A expression levels were calculated relative to $\beta$-actin using the $2^{-\Delta \Delta \mathrm{CT}}$ method.

\section{Western Blotting}

We used a protein extraction kit (KeyGEN Biotech, Nanjing, China). The concentration of the protein was measured using a Bio-Rad Protein Assay Kit (KeyGEN Biotech). The protein sample was subjected to 
electrophoresis and then electrotransferred to a PVDF membrane (Millipore, USA). After completion, the cells were blocked with $5 \%$ skim milk powder in TBST buffer for 1 hour. Then incubate with the corresponding antibody and overnight at $4{ }^{\circ} \mathrm{C}$. The next day, it was incubated with the corresponding secondary antibody for 1 hour at room temperature. Then check the strip condition. This study used $\beta$-actin as an internal reference. In addition, the antibody information used herein is as follows: rabbit anti-KIF2A antibody (1:1, 0000 dilution, ab197988, Abcam plc, Cambridge, UK), rabbit anti- $\beta$ actin (1:1000 dilution, ab8227, Abcam plc, UK) Cambridge), rabbit anti-Ki67 (1:1000 dilution, ab16667, Abcam plc, Cambridge, UK) and rabbit anti-proliferating cell nuclear antigen (PCNA) (1:500 dilution, ab18197, Abcam plc, Cambridge, UK).

\section{Colony Formation Assay}

A total of 500 cells were added to each well and seeded into 6-well plates, followed by incubation for 2 weeks. The resulting colonies were then fixed with methanol and then stained with $0.1 \%$ crystal violet to finally obtain the size and number of colonies.

\section{Cell Counting Kit-8 (CCK-8) Assay}

We used CCK-8 reagent (Beyotime Technology) to measure cell viability. To each well, 2000 cells were added to the 96-well plate, and finally the survival rate was recorded on the third day.

\section{Experiment of Tumor Growth in Mice}

Our institutional animal care committee approved the care and operation of mice. $\mathrm{BalB} / \mathrm{c}$ nude mice (6-8
A
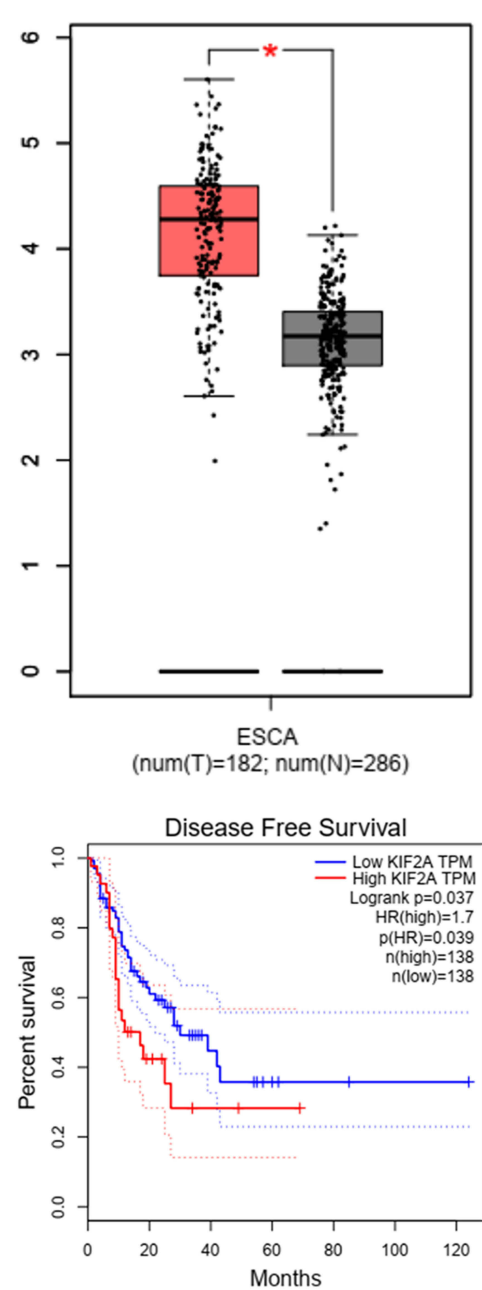

B

100

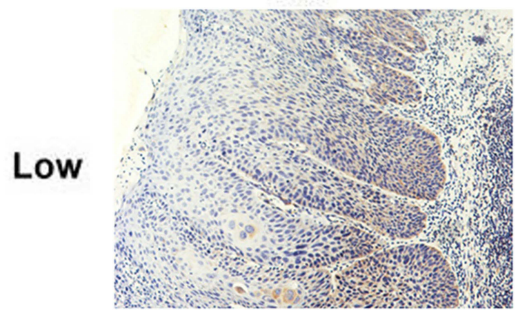

High

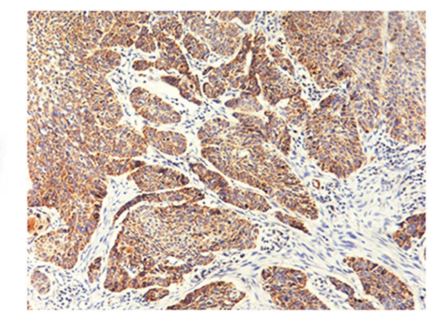

200
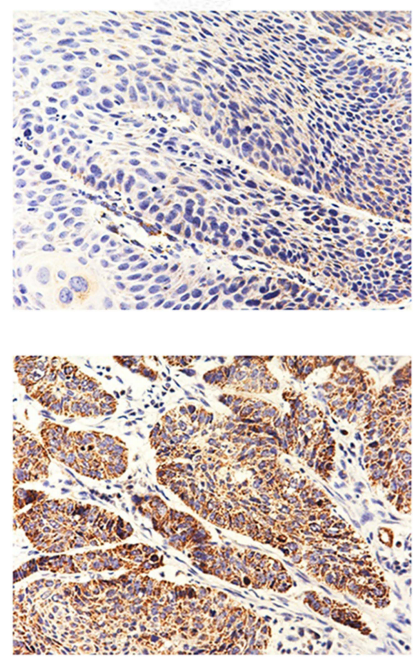

C

100

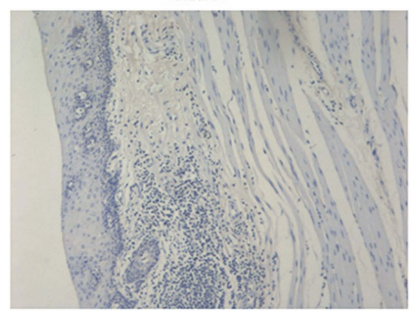

200

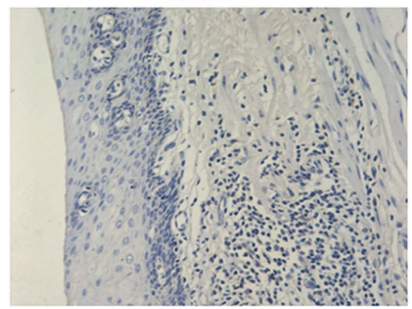

Figure I The expression of KIF2A and the disease-free survival in ESCC. (A) Upper: the expression of KIF2A mRNA in tumor tissues and normal tissues from TCGA. Below: KIF2A overexpression is associated with poor disease-free survival form TCGA. (B) Low and high expressed of KIF2A in tumor tissues. (C) The expression of KIF2A in normal tissues. ${ }^{*} \mathrm{P}<0.05$. 
weeks, 18-22 g) were purchased from Beijing Life River Experimental Animal Technology Co., Ltd. (Beijing, China). First, 6-week-old BALB/c mice were divided into two groups, and KIF2A shRNA or control cells were injected subcutaneously into mice. The volume of the tumor was measured every 7 days for about 2 weeks after the completion of the injection. The tumor is finally isolated and photographed. All applicable international, national, and/or institutional guidelines for the care and use of animals were followed. The animal study was carried out in accordance with the guidelines approved by the Animal Experimentation Ethics Committee of Xingtai people's hospital. The protocol was approved by the Committee of Xingtai people's hospital, all surgery and all efforts were made to minimize suffering.

\section{Statistical Analysis}

The statistical analysis results of this paper were completed by SPSS 23.0 software (IBM, Chicago, Illinois, USA). Statistically significant comparisons were made between the two groups using an independent Student's $t$-test. Data are expressed as mean $\pm \mathrm{SD}, \mathrm{P}<0.05$ is considered statistically significant.

\section{Results}

KIF2A was Overexpressed and Associated with Poor Prognosis in ESCC To investigate the mRNA expression level of KIF2A in ESCC, we obtained ESCC data from the TCGA database for bioinformatics analysis. KIF2A mRNA expression was significantly higher in ESCC specimens $(\mathrm{n}=182)$ compared to normal specimens $(\mathrm{n}=286)(\mathrm{P}<0.001$, Figure 1A). To investigate the relationship between KIF2A expression levels and prognosis in ESCC, we continued to perform bioinformatics analysis of the ESCC dataset in the TCGA database. Kaplan-Meier survival analysis showed that specimens with high KIF2A expression $(n=138)$ had poor disease-free survival (DFS) compared with specimens with low KIF2A expression $(n=138)(H R=1.7, P=0.037$, Figure $1 A)$. Then, we performed IHC in our own samples to detect the protein expression level of KIF2A. The results indicate that the KIF2A protein is highly expressed in most ESCC tissues (Figure 1B). We also divided KIF2A in the ESCC organization into two groups according to the level of expression (Figure 1B). In addition, we rarely see high expression of
KIF2A in normal tissues (Figure $1 \mathrm{C}$ ). We analyzed the relationship between the expression of KIF2A and clinicopathological features. The results showed that the expression of KIF2A in ESCC was significantly correlated with tumor size $(\mathrm{P}=0.003)$ and $\mathrm{T}$ stage $(\mathrm{P}=0.008)$ (Table 1). However, there were no significant differences between the high and low KIF2A groups in other aspects of clinicopathological features, such as age, gender, tumor grade, and lymph node metastasis. These results indicate that KIF2A is highly expressed in ESCC and is significantly associated with poor prognosis.

\section{KIF2A Supports ESCC Proliferation}

\section{in vitro}

To investigate the role of KIF2A in ESCC, we used shRNA vectors to establish CaEs-17 and EC-109 cell lines that knock down KIF2A. The knockdown effect was confirmed by qPCR (Figure 2A) and Western blot (Figure 2B). Among the two short hairpin RNAs we

Table I Relationships of KIF2A and Clinicopathological Characteristics in 78 Patients with Esophageal Cancer

\begin{tabular}{|c|c|c|c|c|c|}
\hline \multirow[t]{3}{*}{ Feature } & \multirow[t]{3}{*}{$\begin{array}{l}\text { All } \\
n=78\end{array}$} & \multicolumn{2}{|c|}{$\begin{array}{l}\text { KIF2A } \\
\text { Expression }\end{array}$} & \multirow[t]{3}{*}{$\chi^{2}$} & \multirow[t]{3}{*}{$P$} \\
\hline & & Low & High & & \\
\hline & & $\mathrm{n}=\mathbf{2 8}$ & $n=50$ & & \\
\hline Age (year) & & & & 2.842 & 0.092 \\
\hline$<60$ & 46 & 13 & 33 & & \\
\hline$\geq 60$ & 32 & 15 & 17 & & \\
\hline Gender & & & & 0.268 & 0.605 \\
\hline Male & 50 & 19 & 31 & & \\
\hline Female & 28 & 9 & 19 & & \\
\hline Tumor grade & & & & 2.457 & 0.117 \\
\hline Low & 48 & 14 & 34 & & \\
\hline High & 30 & 14 & 16 & & \\
\hline Tumor size & & & & 9.018 & $0.003 *$ \\
\hline$<3.5 \mathrm{~cm}$ & 38 & 20 & 18 & & \\
\hline$\geq 3.5 \mathrm{~cm}$ & 40 & 8 & 32 & & \\
\hline T stage & & & & 6.982 & $0.008 *$ \\
\hline $\mathrm{T}_{1-2}$ & 49 & 23 & 26 & & \\
\hline $\mathrm{T}_{3-4}$ & 29 & 5 & 24 & & \\
\hline $\begin{array}{l}\text { Lymph node } \\
\text { metastasis }\end{array}$ & & & & 0.997 & 0.318 \\
\hline Yes & 25 & 7 & 18 & & \\
\hline No & 53 & 21 & 32 & & \\
\hline
\end{tabular}

Note: $* \mathrm{p}<0.05$.

Abbreviations: $\chi^{2}$, chi-square test; $\mathrm{P}, \mathrm{P}$ value. 
A

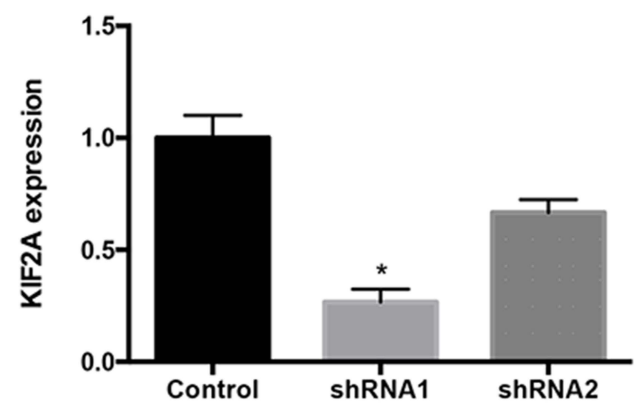

B
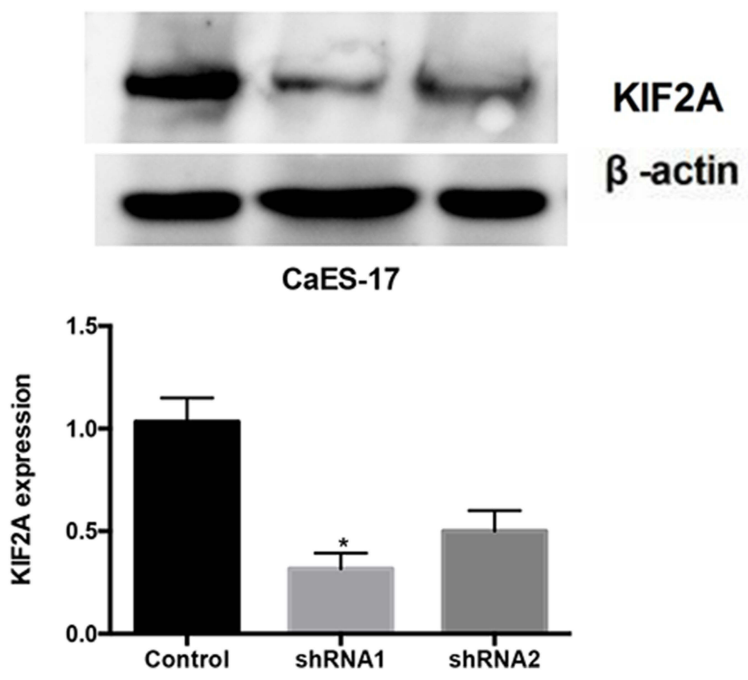

EC-109

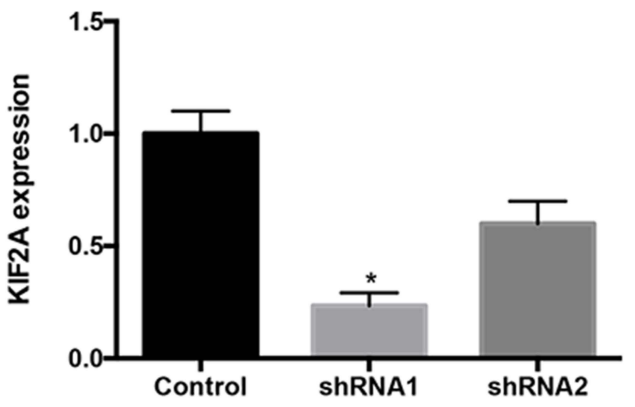

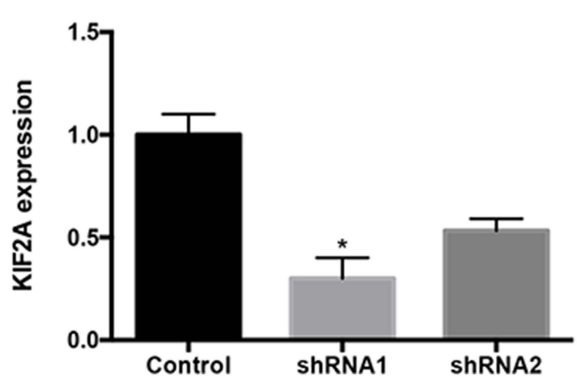

Figure 2 KIF2A knocking down cells for CaEs-17 and EC-I09 cell lines. (A) RT-qPCR showed shRNA knocked down KIF2A in CaEs-17 and EC-I09 cell lines. (B) Upper: Western blotting showed shRNA knocked down KIF2A. Below: Histograms with the gray value of the western booting strips. $* \mathrm{P}<0.05$.

selected, shRNA1 (sh1) showed the most significant knockdown effect, so we chose to use sh1 in the next experiments. We first performed a colony formation test. When knocking down KIF2A, we found that its ability to clone formation was significantly reduced (Figure 3A). We then tested CCK-8 to assess proliferation and viability. When KIF2A was knocked down, viability and proliferation were significantly reduced (Figure 3B). In addition, we also used Western blotting to detect proliferation and selected two proliferative markers Ki67 (Figure 3C) and PCNA (Figure 3D) in CaEs-17 and EC-109 cells. The results showed that $\mathrm{Ki} 67$ and PCNA were significantly decreased in the knockdown group, indicating that proliferation was inhibited after knockdown of KIF2A. These data indicate that KIF2A can significantly promote the proliferation of ESCC cells in vitro.

\section{Downregulation of KIF2A Inhibits Proliferation of ESCC Cells in vivo}

To investigate the effect of KIF2A on ESCC proliferation in vivo, we divided 6-week-old $\mathrm{BALB} / \mathrm{c}$ mice into two groups and subcutaneously injected KIF2A shRNA or shRNA to attenuate lentiviral cells. Measurement of tumor volume was performed every 7 days after about 2 weeks after injection of the cells. The results showed that the tumor volume was significantly smaller than that of the control knockout group (Figure 4A). We then used IHC to detect the expression of KIF2A in mouse tumors. The results showed that KIF2A was significantly lower in the knockdown group than in the control group (Figure 4B). We also used IHC to detect mouse tumor proliferation markers Ki67 and PCNA, and the results showed that both Ki67 and PCNA were significantly reduced in the 
A

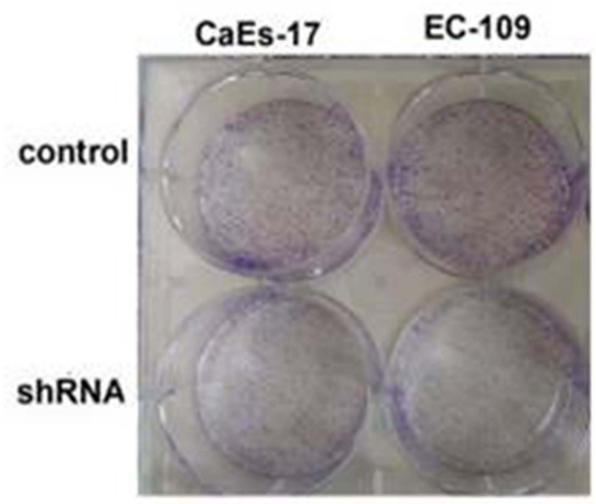

B

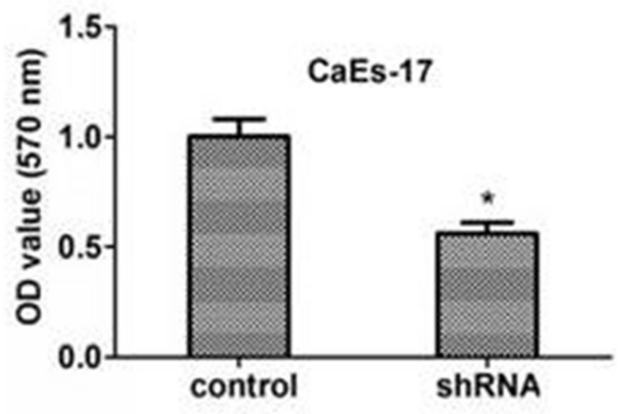

C
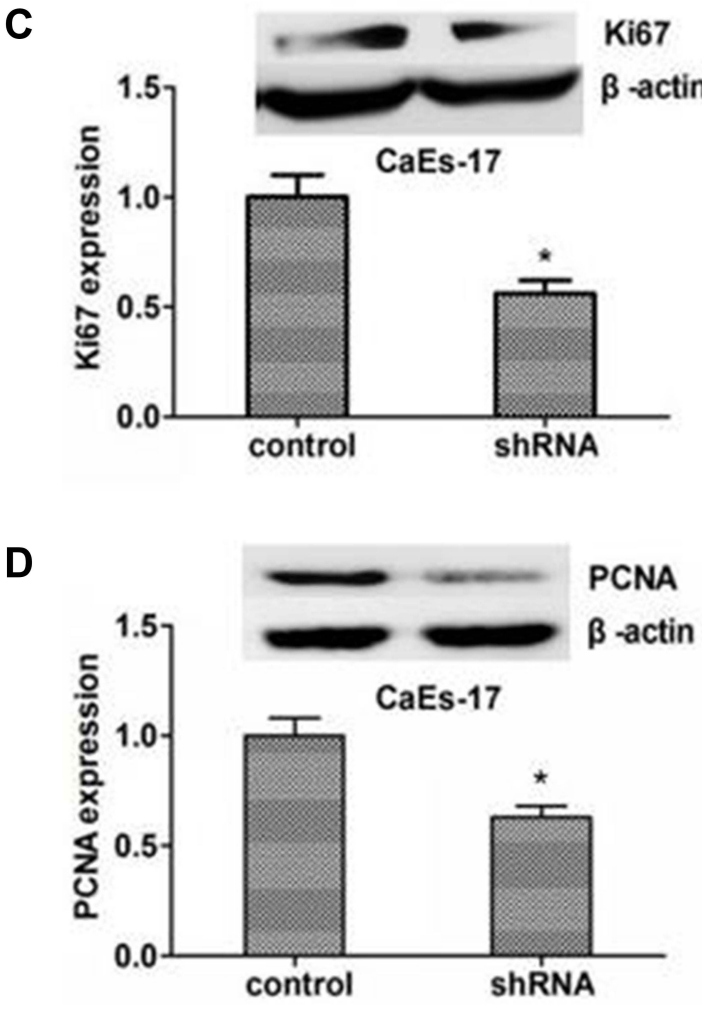
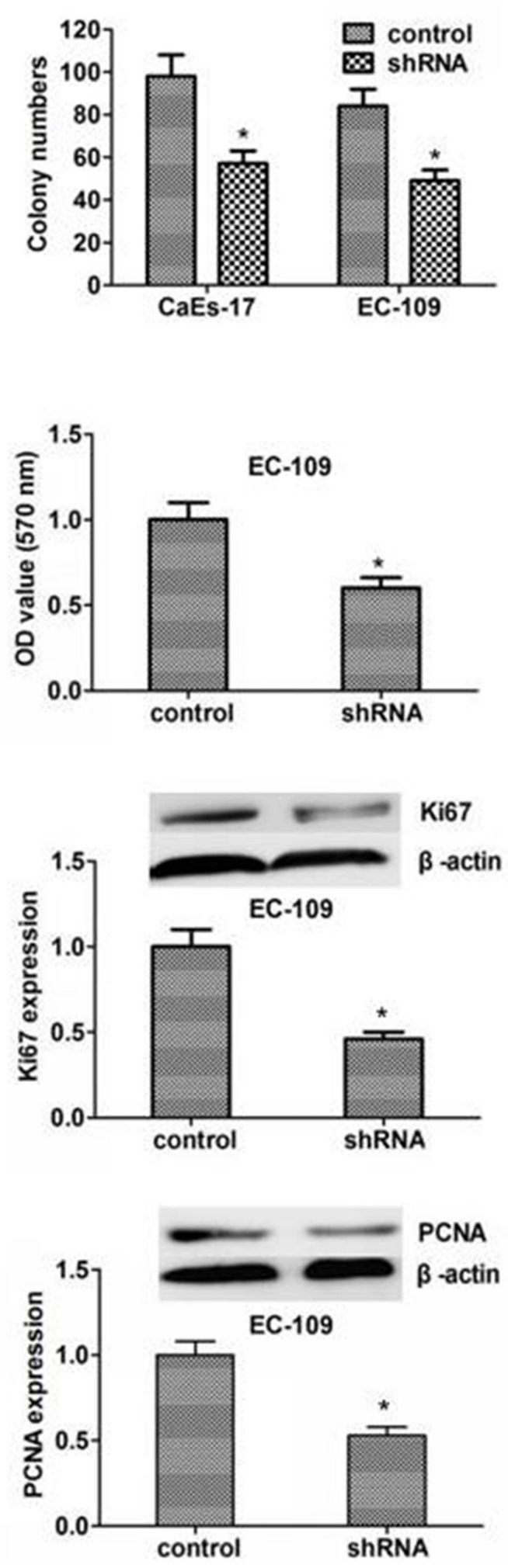

Figure 3 Knocking down of KIF2A can inhibit the proliferation and growth of ESCC cells. (A) Left: clone formation assay to assess the role of KIF2A knocking down in ESCC cells. Right: Histograms with the number of colony. (B) CCK8 to assess the role of KIF2A knocking down in ESCC cells. (C) UPPer: Ki67 to assess the role of KIF2A knocking down for proliferation by Western blotting. Below: histograms with the gray value of the western booting strips. (D) Upper: PCNA to assess the role of KIFCI knocking down for proliferation by Western blotting. Below: histograms with the gray value of the western booting strips. *P<0.05. 
A

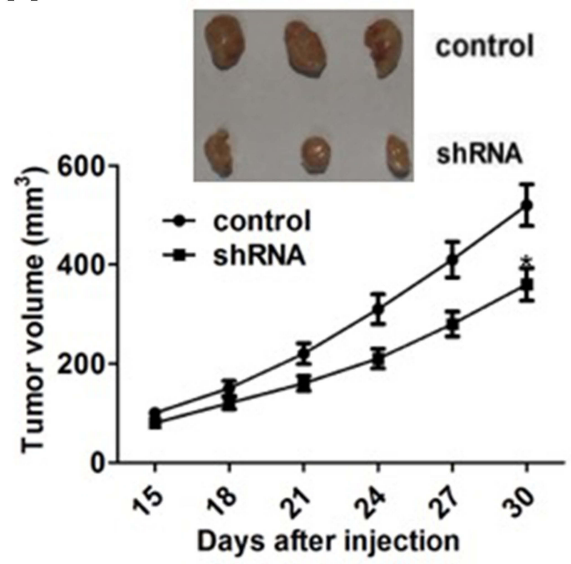

C

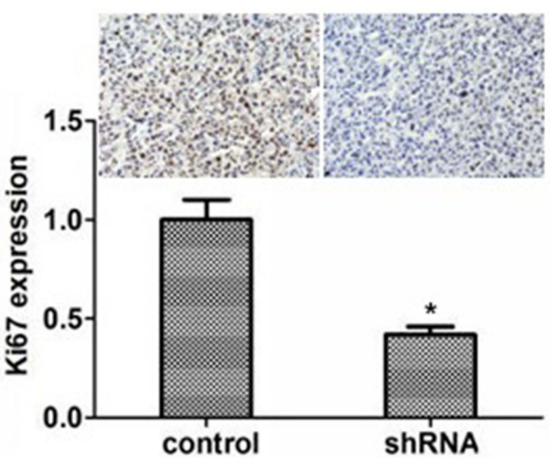

B
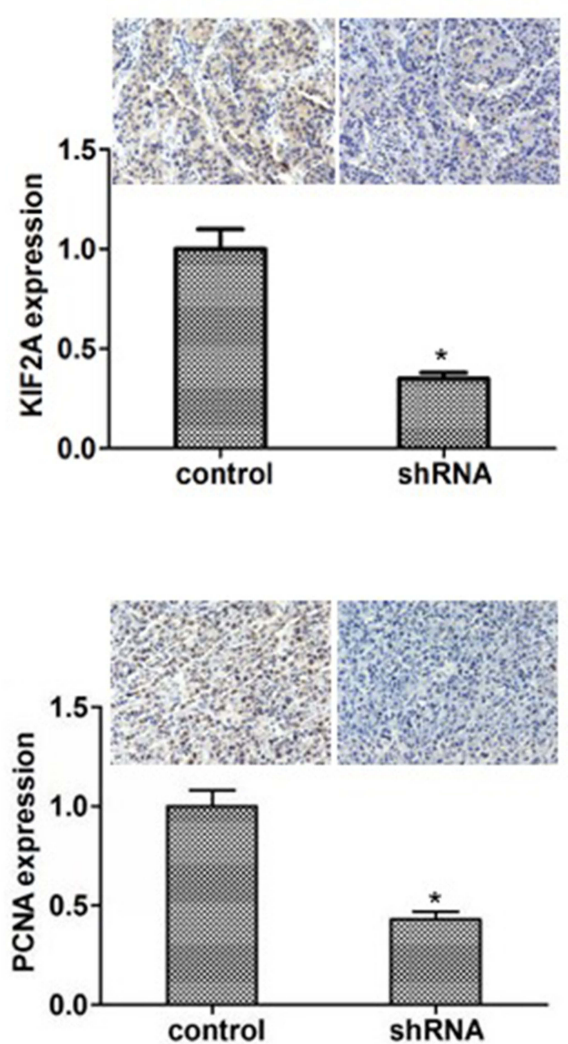

Figure 4 Knocking down of KIF2A can inhibit the proliferation and growth of ESCC cells in vivo. (A) Upper: representative images of the tumors in mice. Below: line chart with volume of tumor in mice. (B) Upper: detection of KIF2A expression in the tumor of mice by IHC. Below: histograms with the gray value of IHC. (C) Upper: representative images of Ki67 and PCNA staining in the tumor of mice. Below: histograms with the gray value of the Ki67 and PCNA staining. $* \mathrm{P}<0.05$.

knockdown group compared to the control group (Figure 4C). These results indicate that KIF2A still promotes the proliferation of ESCC cells in vivo.

\section{Discussion}

$\mathrm{KIF} 2 \mathrm{~A}$ is an important protein for cell mitosis and spindle assembly. ${ }^{18}$ It has also been reported to be a carcinogenic protein that is overexpressed in many human malignancies. ${ }^{10,12-14}$ The high expression level of KIF2A is associated with poor prognosis of gastric cancer, ${ }^{17,20}$ breast cancer $^{15}$ and glioma, ${ }^{18}$ etc. $^{21}$ In our study, KIF2A has been found to be overexpressed in most ESCC patients, and the expression level is correlated with the prognosis of ESCC for DFS. We have reason to believe that KIF2A may have a role in the progression and development of ESCC.

KIF2A is a member of the kinesin-13 family and is a MT depolymerase. ${ }^{22}$ MTs are components of the cytoskeleton that play an important role in mitosis and transport. ${ }^{23}$ In other cancers, it has been reported that KIF2A can promote proliferation. $^{24-26}$ In our study, by establishing two KIF2A knockdown cell line models, the cell proliferation ability of knockout KIF2A was significantly reduced. In in vivo experiments, the results of animal experiments were consistent with cellular experiments. Animal experiments make our conclusions more credible than pure cell experiments.

The study of KIF2A signaling pathways that promote ESCC proliferation is lacking in this paper. Wang et al reported that KIF2A silencing inhibits the phosphatidylinositol 3-kinase (PI3K)/AKT pathway and leads to increased apoptosis. ${ }^{16} \mathrm{Xie}$ et al showed that the expression levels of phosphorylated AKT and phosphorylated ERK1/ 2 in the KIF2A knockout group were significantly reduced. $^{25}$ In addition, the extracellular signal-regulated kinase (ERK) 1/2 pathway is an effector that controls the expression of these kinesins. ${ }^{27,28}$ The Raf/ERK1/2 and PI3K pathways are major factors in Ras transformation and can have a dramatic effect on the cytoskeleton. ${ }^{29,30}$ Sheng et al reported that miR-206 inhibited the overexpression of KIF2A ${ }^{31}$ and Uchida et al showed that the regulation of KIF2A by anti-tumor miR-451a can inhibit 
the aggressiveness of cancer cells. ${ }^{32}$ These studies can play a guiding role in our future analysis of mechanisms.

Our research has some limitations that need to be further improved in future research. First, our prognostic results are mainly from public databases, lacking our own follow-up data. Second, the number of tissue specimens is relatively small, requiring multi-center large sample research support. Third, the depth of mechanism research is insufficient, so more and more in-depth mechanisms should be revealed in the future.

\section{Conclusion}

In conclusion, the results suggested that KIF2A might be a prognostic factor for ESCC. Moreover, KIF2A could promote ESCC proliferation in vitro and in vivo, respectively. These results provided a theoretical basis for the future use of KIF2A as a potential biomarker and therapeutic target for ESCC.

\section{Disclosure}

The authors declare that they have no conflicts of interest regarding this work.

\section{References}

1. Bollschweiler E, Plum P, Monig SP, Holscher AH. Current and future treatment options for esophageal cancer in the elderly. Expert Opin Pharmacother. 2017;18(10):1001-1010. doi:10.1080/14656566.2017. 1334764

2. Sohda M, Kuwano H. Current status and future prospects for esophageal cancer treatment. Ann Thorac Cardiovasc Surg. 2017;23 (1):1-11. doi:10.5761/atcs.ra.16-00162

3. Bray F, Ferlay J, Soerjomataram I, Siegel RL, Torre LA, Jemal A. Global cancer statistics 2018: GLOBOCAN estimates of incidence and mortality worldwide for 36 cancers in 185 countries. CA Cancer J Clin. 2018;68(6):394-424. doi:10.3322/caac.21492

4. Malhotra GK, Yanala U, Ravipati A, Follet M, Vijayakumar M, Are C. Global trends in esophageal cancer. J Surg Oncol. 2017;115 (5):564-579. doi:10.1002/jso.24592

5. Arnold M, Soerjomataram I, Ferlay J, Forman D. Global incidence of oesophageal cancer by histological subtype in 2012. Gut. 2015;64 (3):381-387. doi:10.1136/gutjnl-2014-308124

6. Allum WH, Bonavina L, Cassivi SD, et al. Surgical treatments for esophageal cancers. Ann N Y Acad Sci. 2014;1325:242-268. doi:10.1111/nyas. 12533

7. Buzurovic IM, Hansen JL, Bhagwat MS, et al. Clinical implementation of a novel applicator in high-dose-rate brachytherapy treatment of esophageal cancer. J Contemp Brachytherapy. 2016;8(4):319-325. doi: $10.5114 /$ jcb. 2016.61933

8. van Nistelrooij AM, van Steenbergen LN, Spaander MC, et al. Treatment and outcome of young patients with esophageal cancer in the Netherlands. J Surg Oncol. 2014;109(6):561-566. doi:10.1002/jso.23533

9. Moore A, Wordeman L. The mechanism, function and regulation of depolymerizing kinesins during mitosis. Trends Cell Biol. 2004;14 (10):537-546. doi:10.1016/j.tcb.2004.09.001

10. Manning AL, Ganem NJ, Bakhoum SF, Wagenbach M, Wordeman L, Compton DA. The kinesin-13 proteins Kif2a, Kif2b, and Kif2c/ MCAK have distinct roles during mitosis in human cells. Mol Biol Cell. 2007;18(8):2970-2979. doi:10.1091/mbc.e07-02-0110
11. Ishikawa K, Kamohara Y, Tanaka F, et al. Mitotic centromere-associated kinesin is a novel marker for prognosis and lymph node metastasis in colorectal cancer. Br J Cancer. 2008;98(11):1824-1829. doi:10.1038/sj. bjc. 6604379

12. Kline-Smith SL, Walczak CE. The microtubule-destabilizing kinesin XKCM1 regulates microtubule dynamic instability in cells. Mol Biol Cell. 2002;13(8):2718-2731. doi:10.1091/mbc.e01-12-0143

13. Ganem NJ, Compton DA. The KinI kinesin Kif2a is required for bipolar spindle assembly through a functional relationship with MCAK. J Cell Biol. 2004;166(4):473-478. doi:10.1083/jcb.200404012

14. Wang CQ, Qu X, Zhang XY, et al. Overexpression of Kif2a promotes the progression and metastasis of squamous cell carcinoma of the oral tongue. Oral Oncol. 2010;46(1):65-69. doi:10.1016/j.oraloncology. 2009.11.003

15. Wang J, Ma S, Ma R, et al. KIF2A silencing inhibits the proliferation and migration of breast cancer cells and correlates with unfavorable prognosis in breast cancer. BMC Cancer. 2014;14:461. doi:10.1186/ 1471-2407-14-461

16. Wang K, Lin C, Wang C, et al. Silencing Kif2a induces apoptosis in squamous cell carcinoma of the oral tongue through inhibition of the PI3K/Akt signaling pathway. Mol Med Rep. 2014;9(1):273-278. doi:10.3892/mmr.2013.1804

17. Zhang S, Huang F, Wang Y, Song Q, Yang X, Wu H. KIF2A overexpression and its association with clinicopathologic characteristics and poor prognoses in patients with gastric cancer. Dis Markers. 2016;2016:7484516. doi:10.1155/2016/7484516

18. Zhang X, Ma C, Wang Q, et al. Role of KIF2A in the progression and metastasis of human glioma. Mol Med Rep. 2016;13(2):1781-1787. doi: $10.3892 / \mathrm{mmr} .2015 .4700$

19. Weinstein JN, Collisson EA, Mills GB, et al. The cancer genome atlas pan-cancer analysis project. Nat Genet. 2013;45(10):1113-1120. doi:10.1038/ng.2764

20. Zhao P, Lan F, Zhang H, Zeng G, Liu D. Down-regulation of KIF2A inhibits gastric cancer cell invasion via suppressing MT1-MMP. Clin Exp Pharmacol Physiol. 2018;45(10):1010-1018. doi:10.1111/14401681.12974

21. Fan X, Wang X, Zhu H, Wang W, Zhang S, Wang Z. KIF2A overexpression and its association with clinicopathologic characteristics and unfavorable prognosis in colorectal cancer. Tumour Biol. 2015;36 (11):8895-8902. doi:10.1007/s13277-015-3603-z

22. Miki H, Okada Y, Hirokawa N. Analysis of the kinesin superfamily: insights into structure and function. Trends Cell Biol. 2005;15 (9):467-476. doi:10.1016/j.tcb.2005.07.006

23. Honore S, Pasquier E, Braguer D. Understanding microtubule dynamics for improved cancer therapy. Cell Mol Life Sci. 2005;62 (24):3039-3056. doi:10.1007/s00018-005-5330-x

24. Sun D, Zhou X, Yu HL, et al. Regulation of neural stem cell proliferation and differentiation by Kinesin family member $2 \mathrm{a}$. PLoS One. 2017;12(6):e0179047. doi:10.1371/journal.pone. 0179047

25. Xie T, Li X, Ye F, et al. High KIF2A expression promotes proliferation, migration and predicts poor prognosis in lung adenocarcinoma. Biochem Biophys Res Commun. 2018;497(1):65-72. doi:10.1016/j. bbrc.2018.02.020

26. Broix L, Asselin L, Silva CG, et al. Ciliogenesis and cell cycle alterations contribute to KIF2A-related malformations of cortical development. Hum Mol Genet. 2018;27(2):224-238. doi:10.1093/ $\mathrm{hmg} / \mathrm{ddx} 384$

27. Zaganjor E, Weil LM, Gonzales JX, Minna JD, Cobb MH. Ras transformation uncouples the kinesin-coordinated cellular nutrient response. Proc Natl Acad Sci U S A. 2014;111(29):10568-10573. doi:10.1073/pnas.1411016111

28. Zaganjor E, Osborne JK, Weil LM, et al. Ras regulates kinesin 13 family members to control cell migration pathways in transformed human bronchial epithelial cells. Oncogene. 2014;33(47):5457-5466. doi:10.1038/onc.2013.486 
29. Karnoub AE, Weinberg RA. Ras oncogenes: split personalities. Nat Rev Mol Cell Biol. 2008;9(7):517-531. doi:10.1038/nrm2438

30. Downward J. Targeting RAS signalling pathways in cancer therapy. Nat Rev Cancer. 2003;3(1):11-22. doi:10.1038/nrc969

31. Sheng N, Xu YZ, Xi QH, et al. Overexpression of KIF2A is suppressed by miR-206 and associated with poor prognosis in ovarian cancer. Cell Physiol Biochem. 2018;50(3):810-822. doi:10.1159/000494467
32. Uchida A, Seki N, Mizuno K, et al. Regulation of KIF2A by antitumor miR-451a inhibits cancer cell aggressiveness features in lung squamous cell carcinoma. Cancers. 2019;11(2):258. doi:10.3390/ cancers 11020258

\section{Publish your work in this journal}

Cancer Management and Research is an international, peer-reviewed open access journal focusing on cancer research and the optimal use of preventative and integrated treatment interventions to achieve improved outcomes, enhanced survival and quality of life for the cancer patient.
The manuscript management system is completely online and includes a very quick and fair peer-review system, which is all easy to use. Visit http://www.dovepress.com/testimonials.php to read real quotes from published authors. 